\title{
Vigencia de los aportes de Celso Furtado
} al estructuralismo

\author{
Ricardo Bielschowsky
}

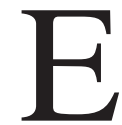

n este trabajo se presentan los tres principales aportes analíticos de Celso Furtado al estructuralismo: i) el método histórico-estructural, que incorpora la historia brasileña y latinoamericana a las formulaciones estructuralistas; ii) el concepto de que el subdesarrollo en la periferia latinoamericana tiende a preservarse por mucho tiempo debido a la dificultad para superar el subempleo y la inadecuada diversificación de la actividad productiva, y iii) la idea de que la evolución de las inversiones en la periferia está predeterminada por la composición de la demanda, que refleja y tiende a mantener la concentración del ingreso y la propiedad. A raíz de lo ocurrido en América Latina en los últimos 25 años, se concluye que el análisis de Furtado tiene hoy plena vigencia. 


\section{I}

\section{Introducción}

En este breve artículo se señalan los principales aportes de Celso Furtado al estructuralismo, o sea, a la teoría de Raúl Prebisch y de la CEPAL sobre el desarrollo periférico. Se trata de aportes analíticos al debate latinoamericano y brasileño sobre crecimiento y desarrollo, que tuvieron importantes repercusiones intelectuales e ideológicas, especialmente en Brasil. El examen de las principales tendencias en ese país y en América Latina entre 1980 y 2005, efectuado a partir de esas contribuciones elaboradas hace casi medio siglo, revela la impresionante vigencia de tales aportes. Lamentablemente, esta vigencia se debe a razones muy desalentadoras: el crecimiento, el empleo y la distribución del ingreso en los últimos 25 años confirmaron el escepticismo de Furtado sobre el desarrollo en ausencia de proyectos nacionales concebidos e implementados en forma adecuada.

\section{II}

\section{Prebisch y el desarrollo económico bajo las condiciones estructurales de la periferia}

Para presentar los aportes de Furtado es necesario referirse brevemente a la teoría de las condiciones periféricas del desarrollo formulada por Prebisch respecto de América Latina. ${ }^{1}$ Esta necesidad obedece a que Furtado fue un seguidor de Prebisch, a que este último fue el fundador del estructuralismo latinoamericano y a que su teoría rara vez se aborda en forma apropiada en la literatura sobre desarrollo económico, ya que en general las referencias se limitan a la tesis del deterioro de la relación de precios del intercambio (los términos de intercambio). Como señalara correctamente Octavio Rodriguez en su libro sobre el pensamiento de la CEPAL, ${ }^{2}$ la teoría de Prebisch constituye todo un cuerpo analítico orientado al examen del subdesarrollo en América Latina.

Según esa teoría, las restricciones al crecimiento están determinadas por las condiciones específicas de

\footnotetext{
$\square$ Este trabajo fue presentado en la sesión especial sobre "Celso Furtado y América Latina y el Caribe. Tendencias y perspectivas" organizada por la CEPAL en la Décima Reunión de la Asociación Económica de América Latina y el Caribe (LACEA) que tuvo lugar en París, en octubre de 2005. El autor agradece a Carlos Mussi y Carlos Aguiar de Medeiros por sus excelentes comentarios a este trabajo, y a Franklin Serrano por el valioso diálogo previo a su elaboración. La responsabilidad por las opiniones aquí expresadas es exclusivamente del autor.

${ }^{1}$ Prebisch (1949) y CEPAL (1950 y 1951).

2 Véase Rodriguez (1981).
}

América Latina como periferia del mundo desarrollado. Prebisch caracteriza a las economías de la región en contraste con las economías "centrales". Argumenta que las diferencias corresponden a condiciones de crecimiento inadecuadas en la periferia, que imponen restricciones al proceso de industrialización y al progreso técnico y que requieren estrategias de crecimiento coordinadas por el Estado, porque en esas condiciones las fuerzas de mercado por sí solas no bastan para hacer viable el crecimiento.

En el cuadro 1 se resumen los principales elementos de la teoría de Prebisch sobre el subdesarrollo latinoamericano y sus problemas, que fue adoptada por Furtado y los demás intelectuales estructuralistas.

Prebisch y la CEPAL se basaron en la identificación de ese conjunto de problemas estructurales de la periferia para construir su análisis del crecimiento, el desarrollo periférico y las relaciones centro-periferia, así como sus tesis fundamentales: deterioro de los términos de intercambio, falta de convergencia entre los ingresos por habitante del centro y de la periferia, desequilibrio estructural de la balanza de pagos, vulnerabilidad externa, brecha de ahorro de divisas y dinámica del proceso de industrialización mediante la sustitución de importaciones.

Ante condiciones tan problemáticas, la planificación y la acción estatal se consideraron fundamentales para sustentar la industrialización y el progreso 
CUADRO 1

América Latina: Síntesis de la formulación estructuralista original

\begin{tabular}{ll}
\hline Características de las economías latinoamericanas & Incidencia en la industrialización y el crecimiento \\
\hline Baja diversidad productiva & $\begin{array}{l}\text { Necesidad de inversiones simultáneas en muchos sectores }- \\
\text { proceso muy exigente en materia de ahorro, inversión y divisas } \\
\text { extranjeras }\end{array}$
\end{tabular}

Especialización en agricultura y minería

Dualidad (o marcada heterogeneidad tecnológica) - coexistencia de sectores de alta productividad y de sectores con abundante ocupación de mano de obra a niveles próximos a los de subsistencia

Institucionalidad inadecuada y falta de capacidad empresarial
Limitada capacidad para generar divisas externas debido a la baja demanda mundial de exportaciones y al deterioro de los términos de intercambio, así como a la fuerte demanda de divisas generada por la elevada elasticidad-ingreso de las importaciones

Baja productividad media y reducido excedente como proporción del ingreso

Baja propensión a ahorrar e invertir, e insuficiente acumulación de capital y progreso técnico (parte del excedente se desperdicia en consumo superfluo e inversiones improductivas)

Fuente: Elaboración propia.

técnico y evitar las tendencias perversas inherentes a esas condiciones. Para Prebisch, la principal tendencia perversa era el desequilibrio estructural de la balanza de pagos; según algunos de sus seguidores, entre ellos Noyola-Vásquez (1957) y Sunkel (1958), había también una tendencia estructural a la inflación. Furtado temía a ambas, pero su principal contribución analítica tuvo que ver con la tendencia a la preservación del subempleo y de la mala distribución del ingreso.

\section{III}

\section{Los aportes de Furtado al estructuralismo}

Furtado hizo tres importantes aportes al cuerpo analítico estructuralista. En primer lugar, incorporó al análisis una perspectiva histórica de largo plazo (1959 a 1970) y mostró que durante siglos, en sucesivos períodos de crecimiento y contracción (en Brasil, los ciclos de la caña de azúcar, la minería y el café), se produjeron y reprodujeron dualidades (o heterogeneidades) económicas y sociales, además de la baja diversidad productiva. Su obra Formación económica de Brasil $^{3}$ fue un bien logrado intento de identificar los elementos históricos en la formación del país que legitiman el uso del estructuralismo y de sus conclusiones en materia de política económica (Bielschowsky, 1995). El objetivo era mostrar que la economía brasileña tenía las características de baja diversidad y dua-

\footnotetext{
3 Véase Furtado (1959).
}

lidad indicadas por Prebisch, de manera que el proceso de industrialización de la década de 1950 pasara a ser entendido como problemático debido a las restricciones "histórico-estructurales" al crecimiento que resultaban de esas características, y que la coordinación estatal pasara a ser entendida como indispensable para superarlas.

En segundo lugar, con el libro Desarrollo y subdesarrollo en América Latina ${ }^{4}$ Furtado inició el debate sobre la dificultad de los sectores urbanos modernos para absorber la fuerza de trabajo masiva que se traslada del campo a las ciudades. Probablemente él fue el primero en plantear la posibilidad de que persistiera a largo plazo el subempleo en América Latina, o de que persistiera a largo plazo la dualidad analizada por

\footnotetext{
${ }^{4}$ Véase Furtado (1961).
} 
Lewis (1954). En consecuencia, fue también pionero al afirmar que el aumento de la productividad en sectores modernos puede coexistir largo tiempo con salarios bajos y mantener la mala distribución secular del ingreso en América Latina. Furtado advirtió que, aunque el crecimiento fuera sostenido, sería difícil absorber la abundante oferta de mano de obra en el contexto de las sociedades latinoamericanas, al plantear la idea de que puede haber crecimiento durante un largo período y a la vez mantenerse el desempleo y el subempleo, la heterogeneidad tecnológica, la concentración del ingreso y la injusticia social.

De hecho, en estudios propios sobre el pensamiento económico brasileño y el de la CEPAL (Bielschowsky, 1995 y 2000, respectivamente), el autor del presente artículo no encontró argumentaciones previas a las de Furtado con ese contenido, por lo que concluye que fue Furtado mismo el que inauguró el debate latinoamericano sobre la relación entre el desarrollo, la determinación de los salarios y la concentración de los ingresos en condiciones de subempleo rural y urbano.

En su libro de 1961, que reúne ensayos escritos en la segunda mitad del decenio de 1950, Furtado probablemente anticipó algunas de las ideas básicas de las teorías de la dependencia debatidas a lo largo de la década de 1960. A su juicio, el modelo de crecimiento latinoamericano corresponde a una de las proyecciones históricas de las economías desarrolladas sobre el resto del mundo, según la cual durante la industrialización de la periferia las empresas extranjeras modernas y sus competidoras locales tienden a compartir el sistema productivo con estructuras arcaicas. Esto lleva a los sistemas periféricos a una nueva forma de economía "dual", que depende en gran medida de métodos de producción inadecuados con respecto a los recursos locales y que es incapaz de superar el subdesarrollo en una porción considerable del sistema productivo.

El tercer gran aporte de Furtado al estructuralismo se produjo algunos años después, ${ }^{5}$ con su profundización del análisis de las relaciones entre crecimiento y distribución del ingreso. Argumentó que la concentración del ingreso y la propiedad predetermina la composición sectorial de la inversión y las opciones tec-

\footnotetext{
5 Véase Furtado (1966, 1968, 1972 y 2000).
}

nológicas, llevando a la fracción moderna de la estructura productiva latinoamericana a una densidad de capital similar a la de los países desarrollados. La tecnología utilizada puede contribuir a mantener el pleno empleo y los salarios altos en estos últimos países, pero es inadecuada en América Latina para absorber la abundante oferta de mano de obra y elevar los salarios de manera sistemática. Ese modelo de inversión supone el mantenimiento del desempleo, los bajos salarios y la concentración del ingreso, lo que a su vez, en un círculo vicioso, fortalece la composición inadecuada de las inversiones. Obsérvese que de nuevo se emplea el método de contrastar la periferia con el centro esta vez para argumentar que, contrariamente a lo que ocurre en los países del centro, los modelos de crecimiento en los países de la periferia tienden a preservar la abundancia de mano de obra e impiden que las mejoras de productividad se reflejen en el ingreso de los trabajadores.

Cabe señalar aquí dos fallas en el análisis de Furtado. En primer lugar, en su libro de 1966 afirmó que en el modelo latinoamericano de crecimiento e industrialización se observaban rendimientos decrecientes a escala, que resultaban en una tendencia al estancamiento. Más adelante debió abandonar esa idea, en virtud de datos concluyentes sobre el marcado crecimiento en la región. En segundo lugar, no consideró la posibilidad de que se agote el excedente de mano de obra como resultado del control de la natalidad y de un crecimiento rápido dentro del modelo distributivo existente. No obstante, nada de lo señalado puede oscurecer el hecho de que Furtado inauguraba, más o menos simultáneamente con Maria da Conceição Tavares y Aníbal Pinto, ${ }^{6}$ el debate brasileño y latinoamericano sobre los modelos de crecimiento y de distribución del ingreso.

En suma, el esquema analítico de Prebisch y Furtado es un análisis histórico-estructural de las persistentes heterogeneidad productiva e insuficiente diversificación de la estructura productiva, y es un análisis de las consecuencias de esas dos características en el crecimiento, el empleo y la distribución del ingreso, que deben tomarse como referencias centrales para formular e instrumentar agendas de reforma y desarrollo.

\footnotetext{
6 Véase Tavares (1964) y Pinto (1965).
} 


\section{IV}

\section{Los aportes analíticos de Furtado y las tendencias económicas actuales de América Latina}

Los datos estadísticos sobre la evolución socioeconómica de la gran mayoría de los países latinoamericanos en los últimos 25 años revelan un desempeño mediocre en términos absolutos y en comparación con los 30 años previos de expansión orientada por el Estado: crecimiento muy inferior del producto interno bruto (PIB) y de la productividad, tasas de inversión mucho menores, más desempleo y subempleo e índices de concentración del ingreso que no disminuyeron, pese al aumento de los gastos sociales.

Nada de eso sorprendió a Celso Furtado, que siempre fue escéptico respecto a la generación de crecimiento y empleo y a la redistribución del ingreso en América Latina, al no haber estrategias de crecimiento conducidas por el Estado. Desde los comienzos del decenio de 1980 sostuvo en diferentes ocasiones que la situación era el resultado de reformas inadecuadas, falta de nuevas estrategias desarrollistas y errores de política en materia de deuda, volatilidad de los capitales y globalización.

Sin embargo, en su crítica Furtado fue solo uno más entre muchos economistas heterodoxos, que siguen la misma línea de pensamiento y creen que persistir en los rumbos dictados por las reformas liberalizadoras hace que las economías latinoamericanas sigan siendo rehenes de un proceso de inversión, empleo y crecimiento bajos, salarios también bajos, pobreza y concentración del ingreso. En este breve ensayo no es posible reseñar las interpretaciones de las corrientes de pensamiento neoliberal y heterodoxo en América Latina, pero sí recalcar que la contribución analítica de Prebisch y Furtado ha sido enriquecedora y valiosa para analizar las tendencias actuales.

Para probar este punto se enfrenta una limitación: ni Prebisch, que falleció en la década de 1980, ni Furtado, que abordó el tema de las tendencias solo en artículos breves, hicieron análisis sistemáticos de la evolución de la economía latinoamericana en los últimos 25 años. Intentando sortear esa limitación, en este ensayo se elaboró una lista de aseveraciones que ambos podrían haber hecho, y para lo cual se tomó como base las teorías que ellos formularon.
Cabe advertir que tales aseveraciones inspiradas en Prebisch y Furtado no significan que se crea en la posibilidad de volver a las políticas de las décadas de 1950, 1960 y 1970 . Ciertamente, habrá que adaptar al nuevo contexto estrategias alternativas a las neoliberales: las economías están abiertas al comercio de bienes y servicios, y las políticas macroeconómicas orientadas al crecimiento están limitadas por la existencia de mercados financieros "salvajes" y por la influencia desestabilizadora de corrientes de capital volátiles y, en muchos países, por grandes deudas internas y externas. Además, los Estados nacionales disponen de recursos relativamente más limitados que antes para poner en práctica agendas de desarrollo. No obstante, nada de eso hace menos valioso el esquema analítico de Prebisch y Furtado para el análisis de las tendencias y perspectivas de América Latina y la formulación de nuevas estrategias de crecimiento.

En lo que se refiere a tendencias económicas en los últimos 25 años, Prebisch o Furtado perfectamente podrían haber hecho las siguientes aseveraciones:

i) El período fue de relativo estancamiento en América Latina. Hubo muchos cambios, algunos positivos - como el fin de la inflación y de los desequilibrios fiscales y el aumento de la productividad en muchos sectores-, pero el resultado económico y social general fue muy desfavorable. Correspondió a la fase contractiva del ciclo largo de industrialización y reprodujo algunas de las principales características de las fases recesivas de ciclos previos (agrícolas y mineros): diversificación inadecuada, heterogeneidad y subempleo, bajos rendimientos del trabajo y concentración del ingreso.

ii) Con pocas excepciones, en la mayoría de los países la política económica y las reformas llevadas a cabo contribuyeron a una relativa desindustrialización, y a la pérdida de eslabones de cadenas productivas y de complementariedades intersectoriales e intrasectoriales en la esfera industrial. $\mathrm{La}$ anterior tendencia a la diversificación productiva se revirtió y hubo una desindustrialización 
prematura (UNCTAD, 2003), anterior a la etapa de formación de sectores con uso intensivo de tecnología y de promoción de un entorno adecuado para un sistema de innovación eficaz. Esto debilitó las bases estructurales del crecimiento.

iii) En el período aumentó la heterogeneidad de sectores, subsectores y empresas. Las empresas más grandes, nacionales y extranjeras, contaron con plantas productivas en la frontera tecnológica, pero la modernidad se difundió de manera precaria y desigual en el sistema económico. La evolución de la heterogeneidad fue determinante, sobre todo por medio del aumento de la proporción de la mano de obra empleada en sectores de baja productividad.

iv) El subempleo y el desempleo explican por qué, a pesar del incremento de los gastos sociales, la distribución del ingreso no cambió. Los ocasionales aumentos de productividad se destinaron primordialmente a lucro $-\mathrm{y}$ en algunos casos a cubrir los salarios de trabajadores altamente calificados - y muy poco a la gran masa de trabajadores con poca calificación.

El conjunto de aseveraciones precedente se puede sintetizar de la siguiente manera: el desempeño mediocre de las economías latinoamericanas en los últimos 25 años se debió en gran medida a la implementación de reformas y políticas equivocadas — tan- to respecto de la asignación de recursos como de asuntos macroeconómicos y financieros- y a la falta de estrategias nacionales de desarrollo. Ese desempeño consolidó una diversificación productiva inadecuada, la heterogeneidad estructural, el desempleo y el subempleo, los bajos salarios, la pobreza y la concentración del ingreso, e hizo que persistieran las brechas en materia de divisas, la vulnerabilidad externa y la disparidad de ingreso por habitante con respecto a las economías desarrolladas.

También en torno al análisis de perspectivas es posible elaborar aseveraciones relevantes inspiradas en Prebisch y Furtado. Por ejemplo, se puede decir que hasta ahora, como en otras fases contractivas observadas a lo largo de 500 años de historia económica de la región (cuando transcurrieron largos períodos antes de que se recuperara el ritmo de crecimiento), no parece haber surgido un nuevo motor de desarrollo. Las preguntas clave son las siguientes: ¿se trata de un estancamiento de largo plazo, que mantendrá la dualidad, la heterogeneidad, la restricción de la balanza de pagos y la "divergencia"? ¿Cuáles son en la actualidad las fuerzas que podrían impulsar la inversión, el progreso técnico y el incremento de la productividad en las economías latinoamericanas? ¿Se vislumbra algún nuevo modelo de crecimiento? ¿Qué relación hay entre las estrategias que apuntan a la prosperidad económica futura, al empleo y a la distribución del ingreso?

\section{Furtado, el estructuralismo y el} neoestructuralismo

Aceptar que el conjunto de aseveraciones precedente es importante para entender las tendencias en los últimos 25 años supone decir que el enfoque PrebischFurtado es pertinente y actual.

Entre las mejores interpretaciones heterodoxas de las tendencias actuales en América Latina se encuentran algunos buenos análisis de temas monetarios y financieros enmarcados en el neoestructuralismo cepalino $^{7}$ y corrientes afines. Esos textos constituye-

\footnotetext{
${ }^{7}$ Esta expresión fue acuñada después de que los economistas de la CEPAL asimilaran la irreversibilidad de las reformas liberalizadoras, cuyas fallas criticaron, y realizaran recomendaciones para corregir el
}

ron un avance necesario respecto de las interpretaciones estructuralistas iniciales, sobre todo en la presente era de incertidumbres macroeconómicas, volatilidad del capital y problemas financieros. ${ }^{8}$

En los niveles mesoeconómicos y microeconómicos, la capacidad analítica de los neoestructuralistas

rumbo. El documento considerado inaugural de la etapa neoestructuralista - Transformación productiva con equidad (CEPAL, 1990)fue coordinado por Fernando Fajnzylber, quien transmitió a la CEPAL el pensamiento que había venido desarrollando en los años anteriores (véase Fajnzylber, 1989).

${ }^{8}$ Véase, por ejemplo, CEPAL (1995 y 2002); Ffrench Davis (1999), y Ocampo, Bajraj y Martin (2001). 
de hoy hace justicia a los fundadores de esa corriente. En las publicaciones actuales se señala adecuadamente la necesidad de promover infraestructura e inversiones con uso intensivo de tecnología, sistemas nacionales de innovación y educación, complementariedades sectoriales, economías de aglomeración y pequeñas y medianas empresas.

No obstante, si se comparan los trabajos de los neoestructuralistas actuales con los de sus precursores, se observa que en los de hoy son insuficientes los análisis sobre los factores determinantes de la inversión y sobre los modelos de crecimiento.

La acumulación de capital fue una obsesión de los estructuralistas y de Furtado, lamentablemente descuidada en la actualidad. El análisis neoestructuralista del tema es adecuado porque relaciona la escasez de inversiones con incertidumbres macroeconómicas, entre ellas la volatilidad del capital, la inestabilidad de los tipos de cambio y las tasas de interés, y la contracción y oscilación de los niveles de actividad; pero poco se ha dicho sobre la relación entre la tendencia a invertir y las reformas (liberalización comercial y privatización) y acerca de la propensión a invertir en general.

En uno de los pocos estudios sobre el tema, Moguillansky y Bielschowsky (2001) argumentaron que, con la apertura comercial, las empresas nacionales en los sectores de bienes transables pasaron a tener menos rentabilidad y mayores incertidumbres y riesgos que suponían menores incentivos para invertir. Del mismo modo, antes de las privatizaciones las empresas estatales invertían con menos preocupación por la rentabilidad y con menor aversión a riesgos e incertidumbres. Esto lleva a pensar que las privatizaciones reducen la tendencia a invertir en la economía, aunque tal vez puedan aumentar la eficiencia microeconómica de las inversiones. Hay, por lo tanto, razones para creer que la baja tendencia actual a invertir puede estar relacionada con las reformas y que en el nuevo contexto se requiere coordinación público-privada para favorecer el aumento de las inversiones.
Por último, el neoestructuralismo está en deuda con el estructuralismo original en cuanto a modelos de crecimiento alternativos al neoliberal que tengan en cuenta las condiciones propias de cada país latinoamericano. Mucho se ganaría si se volviera a formular proyectos de desarrollo nacionales siguiendo la línea de pensamiento de Furtado, es decir, la del padrón o modelo de crecimiento que otros — como Aníbal Pinto, Conceição Tavares y Jose Serra, y Pedro Sainz y Alfredo Calcagno_— 9 en ocasiones llamaron "modelos", "estilos" o "modalidades" de crecimiento.

Desde un enfoque histórico-cultural, el modelo de crecimiento es la combinación - determinada históricamente y específica para cada país - de una serie de elementos estructurales, responsables de la dinámica del crecimiento, la inversión, la productividad, el empleo y los salarios. Entre esos elementos cabe destacar los siguientes: i) los principales agentes de inversión (Estado, capital nacional y capital extranjero); ii) la ecuación financiera de la inversión (utilidades retenidas versus financiamiento local e internacional); iii) la composición de la producción y del comercio externo y las opciones tecnológicas; iv) la dirección del crecimiento (hacia adentro, hacia afuera o en ambos sentidos); v) la evolución del empleo y del subempleo, y vi) la evolución de la distribución del ingreso y de la propiedad.

Si a este conjunto de elementos se suman el régimen macroeconómico, las relaciones financieras con el resto del mundo y la institucionalidad existente en cada país, se llega a una buena agenda para el estudio de lo que está ocurriendo en cada economía latinoamericana. Este ejercicio, que sería un excelente punto de partida para trazar un mapa de semejanzas y diferencias en el comportamiento de los diversos países de América Latina, puede ayudar a la formulación de proyectos de desarrollo en cada uno de ellos y a construir una agenda estratégica amplia y diversificada para la región en general.

\footnotetext{
${ }^{9}$ Véase Pinto (1976); Tavares y Serra (1972), y Sainz y Calcagno
} (1992). 


\section{VI}

\section{Resumen y conclusión}

En este breve artículo se presentaron los tres principales aportes analíticos de Celso Furtado al estructuralismo.

El primero, base del método histórico-estructural, fue la incorporación de la historia brasileña y latinoamericana a las formulaciones estructuralistas. El segundo fue el concepto de que el subdesarrollo en la periferia latinoamericana tiende a mantenerse por mucho tiempo debido a la dificultad para superar sus dos condiciones básicas: el subempleo y la inadecuada diversificación de la actividad productiva. De acuerdo con Furtado, el subdesarrollo no es una simple fase de transición al desarrollo, sino un fenómeno más permanente, cuya superación exige una dedicación política tenaz y prolongada.

El tercer aporte consistió en la idea de que la evolución de las inversiones en la periferia está predeterminada por la composición de la demanda, que refleja la concentración del ingreso y de la propiedad.
En América Latina se reproducen estructuras de oferta poco apropiadas para la absorción de mano de obra abundante, que suponen el mantenimiento de los bajos salarios y la tendencia a que se refuerce la desigual distribución del ingreso.

A la luz de lo ocurrido en América Latina en los últimos 25 años, el análisis de Furtado lamentablemente sigue teniendo plena vigencia. Los dos elementos centrales del subdesarrollo - es decir, la insuficiente diversidad de la base productiva y la dualidad o heterogeneidad estructural — no han sido superados, de modo que se han mantenido los bajos salarios, la concentración del ingreso y los elevados índices de pobreza.

Indudablemente, una agenda inspirada en Celso Furtado para la investigación de las tendencias y perspectivas actuales en América Latina sería amplia y necesaria, y confirmaría la vigencia e importancia de los aportes que él hizo. ${ }^{10}$

(Traducido del portugués)

\section{Bibliografía}

Bielschowsky, R. (1995): Pensamento econômico brasileiro. O ciclo ideológico do desenvolvimentismo, Rio de Janeiro, Contraponto.

(2000) (org.): Cinqüenta anos de pensamento na CEPAL: uma resenha, Rio de Janeiro, Record.

Brasil (2003): Plano plurianual 2004-2007, Brasilia, Ministério do Planejamento.

CEPAL (Comisión Económica para América Latina y el Caribe) (1950): Estudio económico de América Latina, 1949, E/CN.12/ 164/rev.1, Santiago de Chile.

(1951): Estudio económico de América Latina, 1950, E/CN.12/217/Rev.1, Santiago de Chile.

(1990): Transformación productiva con equidad: la tarea prioritaria del desarrollo de América Latina y el Caribe en los años noventa, Santiago de Chile, LC/G.1601-P, Santiago de Chile, marzo.

(1995): América Latina y el Caribe: políticas para mejorar la inserción en la economía mundial, LC/G.1800/Rev.1-P), Santiago de Chile. Publicación de las Naciones Unidas, $\mathrm{N}^{\mathrm{o}}$ de venta: S.95.II.G.6.

(2002): Globalización y desarrollo, LC/G.2157(SES.29/3), Santiago de Chile, abril.

Fajnzylber, F. (1989): Industrialización en América Latina: de la "caja negra" al "casillero vacío", serie Cuadernos de la CEPAL, No 60, LC/G.1534/Rev.1-P, Santiago de Chile. Publicación de las Naciones Unidas, $\mathrm{N}^{\circ}$ de venta: S.89.II.G.5.

Ffrench Davis, R. (1999): Macroeconomía, comercio y finanzas. para reformar las reformas en América Latina, Santiago de Chile, CEPAL/McGraw-Hill.
Furtado, C. (1959): Formacão econômica do Brasil, Rio de Janeiro, Fundo de Cultura. (1961): Desarrollo y subdesarrollo, Rio de Janeiro, Fundo de Cultura.

(1966): Subdesenvolvimento e estagnação da América Latina, Rio de Janeiro, Civilização Brasileira. (1968): Um projeto para o Brasil, Rio de Janeiro, Saga. (1972): Análise do "modelo" brasileiro, Rio de Janeiro,

Fundo de Cultura.

(1979): Formação econômica da América Latina, Rio de Janeiro, Cia Editora Nacional.

(2000): Teoria e política do desenvolvimento econômico,

Rio de Janeiro, Paz e Terra.

\footnotetext{
${ }^{10}$ Cabe señalar que en el caso de Brasil el enfoque de Furtado constituye mucho más que una agenda de investigación y está presente como proyecto nacional de desarrollo en el Plan Plurianual 2004-2007 (Brasil, 2003). Aunque este plan fue firmado por el Presidente de la República y aprobado por el Congreso, lamentablemente no parece haber sido adoptado, difundido y seguido adecuadamente por el gobierno. No obstante, en él se define una alentadora estrategia de desarrollo de largo plazo cuyos orígenes conceptuales están precisamente en los trabajos de Furtado de las décadas de 1960 y 1970, en que reclama la formación de un círculo virtuoso de inversión, salarios y consumo popular.
} 
Lewis, W. (1954): Economic development with unlimited supplies of labour, Manchester School of Economic and Social Studies, vol. 22, Manchester, The Manchester School of Economic and Social Studies.

Moguillansky, G. y R. Bielschowsky (2001): Inversión y reformas económicas en América Latina, Santiago de Chile, Comisión Económica para América Latina y el Caribe (CEPAL)/Fondo de Cultura Económica.

Noyola-Vásquez, J. (1957): Inflación y desarrollo económico en México y Chile, Panorama económico, $\mathrm{N}^{\mathrm{o}} 170$, Santiago de Chile, julio.

Ocampo, J.A., R. Bajraj y J. Martin (coords.) (2001): Una década de luces y sombras. América Latina y el Caribe en los años noventa, Santafé de Bogotá, CEPAL/Alfaomega.

Pinto, A. (1965): Concentración del progreso técnico y de sus frutos en el desarrollo de América Latina, El trimestre económico, $\mathrm{N}^{\circ} 125$, México, D.F., Fondo de Cultura Económica.

(1970): Naturaleza e implicaciones de la 'heterogeneidad estructural' de la América Latina, El trimestre económico, $\mathrm{N}^{\circ}$ 145 , enero-marzo.

(1976): Notas sobre los estilos de desarrollo en América Latina, Revista de la CEPAL, $\mathrm{N}^{\circ}$ 1, Santiago de Chile.

Prebisch, R. (1949): El desarrollo económico de América Latina y sus principales problemas (E/CN.12/89), Santiago de Chile,
Comisión Económica para América Latina y el Caribe (CEPAL).

Rodriguez, O. (1981): Teoria do subdesenvolvimento da CEPAL, Rio de Janeiro, Forense/ Universitária.

Sunkel, O. (1958): La inflación chilena, un enfoque heterodoxo, $E$ trimestre económico, vol. 25(4), No 100, México, D.F., Fondo de Cultura Económica, octubre-diciembre.

Sainz, P. y A. Calcagno (1992): En busca de otra modalidad de desarrollo, Revista de la CEPAL, $\mathrm{N}^{\circ}$ 48, LC/G.1748-P, Santiago de Chile, diciembre.

Tavares, M.C. (1964): Auge y declinación del proceso de sustitución de importaciones en el Brasil, Boletín económico de América Latina, vol. 9, $\mathrm{N}^{\mathrm{o}}$ 1, Santiago de Chile, Comisión Económica de América Latina y el Caribe, marzo.

(1979): Auge e declínio da substituição de importações, Da substituição de importações ao capitalismo financeiro, Rio de Janeiro, Zahar.

Tavares, M.C. y J. Serra (1972): Além da estagnação, Da substituição de importações ao capitalismo financeiro, Rio de Janeiro, Zahar.

UNCTAD (Conferencia de las Naciones Unidas sobre Comercio y Desarrollo) (2003): Informe sobre las inversiones en el mundo, Ginebra, Naciones Unidas. 